\title{
Precise Viscosity and Density Sensing in Industrial and Automotive Applications
}

\author{
Thomas Voglhuber-Brunnmaier ${ }^{1}$, Alexander O. Niedermayer ${ }^{2}$, Friedrich Feichtinger ${ }^{1}$, Bernhard Jakoby ${ }^{1}$ \\ ${ }^{1}$ Johannes Kepler University, Altenbergerstr. 69, 4040 Linz, Austria \\ ${ }^{2}$ Micro Resonant Technologies, Hafenstr. 47-51, 4020 Linz, Austria
}

\begin{abstract}
A resonant sensor based system for measuring viscosity and density is shown and applied to two measurement tasks comprising oil characterization in hydraulic systems and measurement of diesel and soot contamination in engine oil. It is demonstrated that from measuring over varying temperature and pressure, a variety of additional physical properties can me exploited for online condition monitoring. This encompasses also the heat expansion coefficient and the bulk modulus, which are difficult to measure otherwise.
\end{abstract}

Keywords: condition monitoring, resonant sensor, bulk modulus, engine oil dilution

\section{Introduction}

Besides the measurement of liquids in production processes, sensors for monitoring lubricating liquid properties are used in order to retrieve information on the condition of the lubricant and the machinery. With such condition monitoring processes, it is possible to shift maintenance policies from a scheduled towards a predictive maintenance. Doing this reduces the maintenance costs by minimizing machine shutdowns for inspection and service, and furthermore, significantly reduces the risk of catastrophic component failure during regular operation and resulting repair downtime [1].

The traditional approach of analyzing oil samples in a laboratory on regular schedule typically gives reliable results but implies a lag in information due to long sampling intervals, shipping and processing time and furthermore, possible irregularities in taking or handling the sample. Another aspect of the bottle sampling approach that is hardly considered is that the sampling schedule often is not synchronized with the duty cycles on the assets, resulting in systematic errors due to violation of the Nyquist-Shannon theorem. Online sensors for monitoring oil properties are capable of increasing the frequency of the tests significantly while reducing total cost of ownership. Furthermore, quick and adequate action can be taken in case of rapid changes due to failures (like water leaking into the oil system).
Compared to offline analyses in a lab, online sensors can only determine a rather limited number of different parameters. That is one of the reasons why some manufacturers try to overcome this drawback by implementing data based oil-ageing models that are configured with reference data from experiments with the very same oil. The data that is provided by such sensors may yield good results in applications where the type of oil is precisely known and the operating conditions of the machinery do not differ from that of the test machines. Under these circumstances the reliability of the condition monitoring is mainly determined by the training data; hence, the confidence of information obtained with this type of sensors will be relatively poor with respect to unexpected changes in the fluid (i.e. caused by failure of components, contamination with alien substances or erroneous refill of wrong fluid).

With respect to the claim that online condition monitoring should increase the reliability of an asset, the confidence of the sensor data as well as the reliability of the condition monitoring system are crucial parameters. Unreliable sensors may provide inconsistent data that trigger false alarms, resulting in unnecessary troubleshooting or even shutdowns and, if occurring frequently, may very likely lead to personnel ignoring alarms. Ultimately, this may result in even higher costs than without any online condition monitoring system at all [2]. 
The measurement system shown in this contribution fulfills stringent requirements in measurement precision and long-term stability. A high level of confidence can be achieved by implementing methods to constantly check sensor data against physical models of liquid properties, such as temperature dependence of viscosity and density, for example.

\section{Measurement Setup}

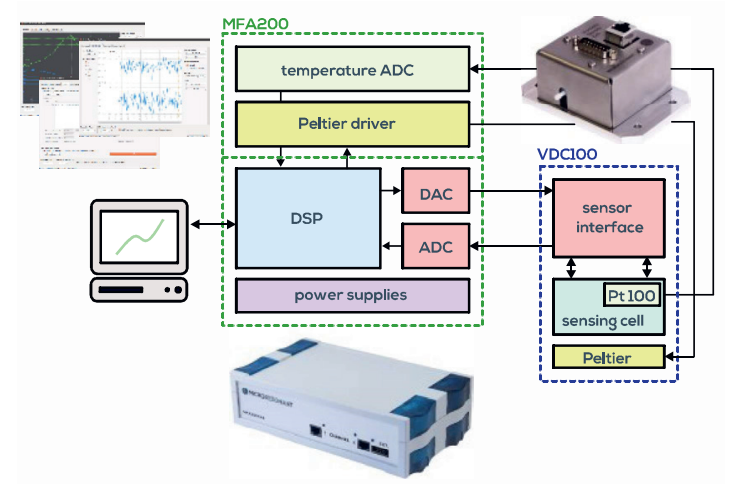

Fig. 1: $\quad$ The measurement setup consists of the temperature controlled measurement cell VDC100 and the universal resonance analyzer MFA200.

The measurement system that was used for the experiments shown in this contribution comprises a flow-through measurement cell VDC100 in combination with the universal resonance analyzer MFA200. Measured viscosity and density parameters are sent to a PC and analyzed in real-time (see Fig. 1). The system was developed by the University Spin-off company Micro Resonant in collaboration with the Institute for Microelectronics and Microsensors at the Johannes Kepler University.

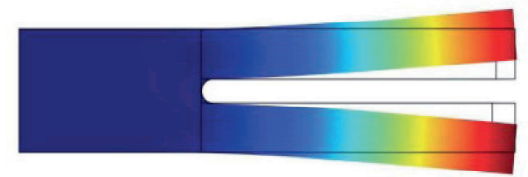

Fig. 2: Fundamental vibration mode of the quartz tuning fork (QTF) used for measurement.

The temperature of the sample volume in the cell is controlled with a thermoelectric element (Peltier) and a PT100 resistive temperature sensor. The physical liquid properties are determined using a piezoelectric quartz tuning fork (QTF) resonator as sensor. In order to obtain the resonance characteristics (i.e. resonance frequency and the $Q$-factor) of the sensor, the QTF is excited with a digitally generated waveform. The frequency components in this waveform are chosen such that the information gathered from the resonator at these frequencies is maximized. This approach enables the use of dedicated signal processing algorithms for suppressing unwanted influences (like offset signals and gain errors) of the analog signal path [3]. For the QTF the Butterworth-Van Dyke equivalent circuit is a usable approximation. The shunt capacitance $C_{0}$ is formed by the quartz material and the electrodes, and the RLC series network represents the electromechanical interaction and is termed motional branch. We consider only the fundamental vibration model shown in Fig. 2, but in principle for each mode of vibration, another set of component values would be required.

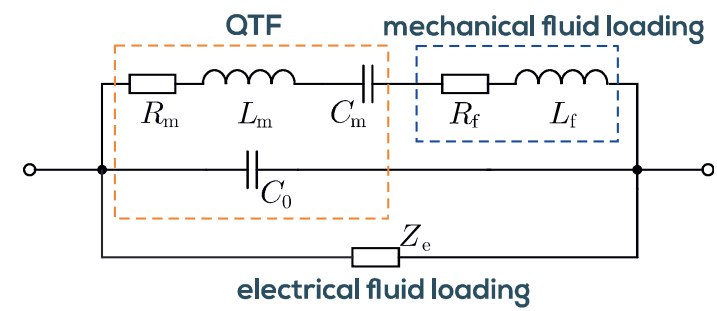

Fig. 3: The extended Butterworth-Van Dyke model describing the fluid loaded quartz tuning fork.

When the QTF vibrates in liquid, the prongs of the QTF periodically displace fluid mass, and therefore, the liquid density adds mass to the equivalent mass, which is reflected in the additional inductance $L_{f}$. The dissipated energy due to viscous drag is accounted for by a resistor $R_{\mathrm{f}}$ in series to the motional branch. The permittivity and conductivity of the fluid also influence the measured impedance. If highly conductive fluids are measured, an insulating layer must be applied to avoid high shunt currents which would reduce the signal-to-noise ratio. This layer, as well as electrochemical double layer effects are summarized in the impedance $Z_{\text {e }}$. The effects of $C_{0}$ and $Z_{e}$ are separated from the measured impedance spectra by the signal processing in the resonance analyzer instrument shown in Fig. 1. Only the resonance parameters of the extended motional branch are analyzed, which enhances the stability of and accuracy of the density and viscosity measurement substantially.

\section{Advantages of using tuning fork sensors}

Compared to alternative viscosity sensors, e.g., based on thickness shear mode resonators, shear horizontal or Love wave sensors (see 
e.g., [4]), using a tuning fork has distinct advantages which are relevant especially in field applications. Due to the low operation frequency of the QTF (here: $25-30 \mathrm{kHz}$ ), the results are more comparable to laboratory instruments, because viscoelastic effects of fluids are less pronounced [5]. The penetration depth of shear waves scale with $f^{-1 / 2}$ and is therefore larger at low frequencies. Furthermore, the penetration depth $\delta$, which usually limits the spatial sensing range of pure shear sensors [6], is not a limiting factor when using QTFs. Given the vibration mode in Fig. 2, the flow profile mainly consists of a potential flow field due to the fluid displacement. The spatial decay of the flow field of the QTF follows approximately $r^{-2}$ instead of $\exp (-r / \delta)$ of a plane shear wave. As was demonstrated in [7], watery suspensions containing spherical particles of $40 \mu \mathrm{m}$ diameter can be measured equally well as smaller particles or clear fluids, although $\delta$ is $3.4 \mu \mathrm{m}$ and therefore much less than the particle size. Consequently, a good spatial average is obtained when measuring fluids with certain microstructure such as emulsions (see e.g. [8]) or suspensions, giving results closer to larger laboratory instruments. Furthermore, also the impact of surface contaminations due to oil additives, soot, or air microbubbles is reduced significantly. Another benefit of QTF resonators is the fact, that viscosity and density can be measured simultaneously. When using pure shear wave sensors only the viscosity-density product can be measured (except by implementing a more complex design such as adding structured surfaces [9]).

\section{Condition Monitoring in Industrial Applications}

The viscosity value of an oil used in a hydraulic or lubricating system is one of the dominant design parameter and therefore, monitoring oil condition by evaluating viscosity is an advantageous approach. Furthermore, viscosity reacts to contamination very sensitively, as is shown in $[10,11]$, where an engine-oil dilution with $0.05 \%$ diesel is reliably detected within seconds. In the described application, the density and viscosity functions of hydraulic oil versus temperature and pressure are measured and additional physical monitoring parameters are derived from the characteristics.

Fig. 4 shows the viscosity of a hydraulic oil versus temperature at various pressures measured with the system in Fig. 1. The high sensitivity of viscosity to temperature is apparent and underpins the need for an accurate temperature control. The temperature characteristics as a new monitoring parameter can be is obtained by measuring at various defined temperatures.

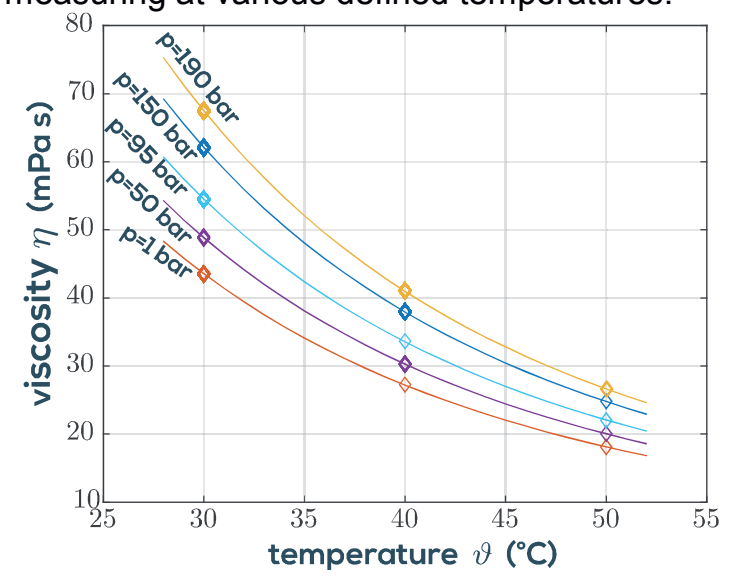

Fig. 4: Viscosity of hydraulic oil over temperature for various pressures.

The solid lines in Fig. 4 follow the Vogel-Cameron Model (DIN 53017) [12] which is given by

$$
\eta(\vartheta)=A e^{\left(\frac{B}{\vartheta+C}\right)}
$$

where $\vartheta$ is the temperature in ${ }^{\circ} \mathrm{C}, C=95^{\circ} \mathrm{C}$ for mineral oils and $A$ and $B$ are fluid specific constants. Besides the strong temperature dependence, liquid viscosity is influenced by pressure. The sensitivity of viscosity to pressure is demonstrated in Fig. 5. Here, the measurement cell (equipped with Minimess connectors) was implemented in industrial environment at a high pressure supply rail of an operating machine.

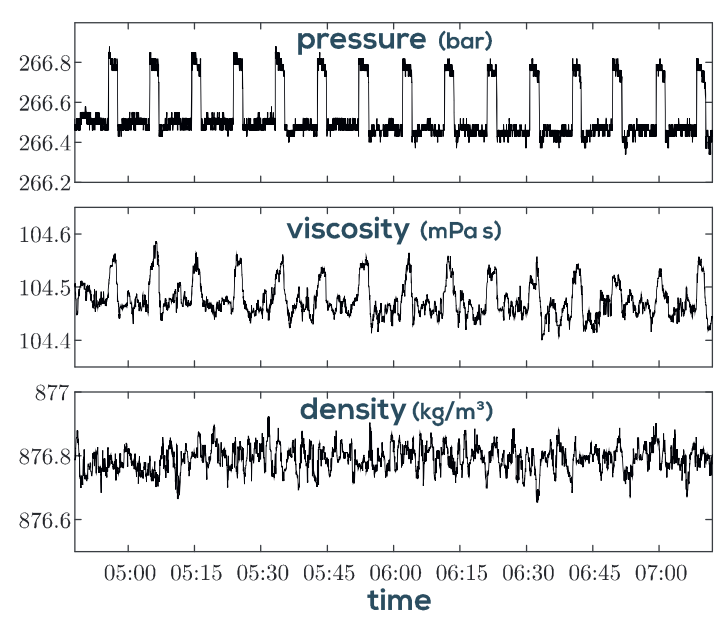

Fig. 5: Small pressure pulses influence viscosity noticeably, but not density.

The cyclic pressure drops of 0.3 bar at 266 bar caused by that machine are clearly visible in the 
viscosity. The viscosity versus pressure is shown in Fig. 6. The dashed lines follow the exponential law given in Eq. (2) with the fitting parameters in Tab. 1.

$$
\eta(p)=\eta_{0} e^{\alpha p}
$$

Here, $\eta_{0}$ is the viscosity at ambient pressure and the viscosity-pressure coefficient $\alpha$ is an additional monitoring parameter.

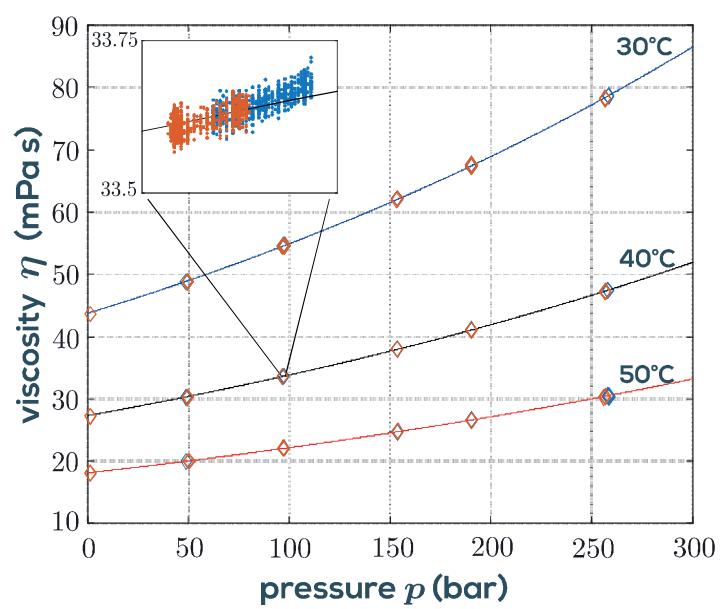

Fig. 6: Viscosity of hydraulic oil over pressure at three different temperatures. The inset shows the noises of two measurement cycles performed at separate days.

Tab. 1: Calculated ambient viscosity and viscosity-pressure coefficient following Eq. (2).

\begin{tabular}{|c|c|c|}
\hline$\vartheta\left({ }^{\circ} \mathrm{C}\right)$ & $\eta_{0}$ (mPas) & $\alpha\left(10^{-3} /\right.$ bar $)$ \\
\hline 30 & 43.75 & 2.272 \\
\hline 40 & 27.33 & 2.139 \\
\hline 50 & 18.14 & 2.016 \\
\hline
\end{tabular}

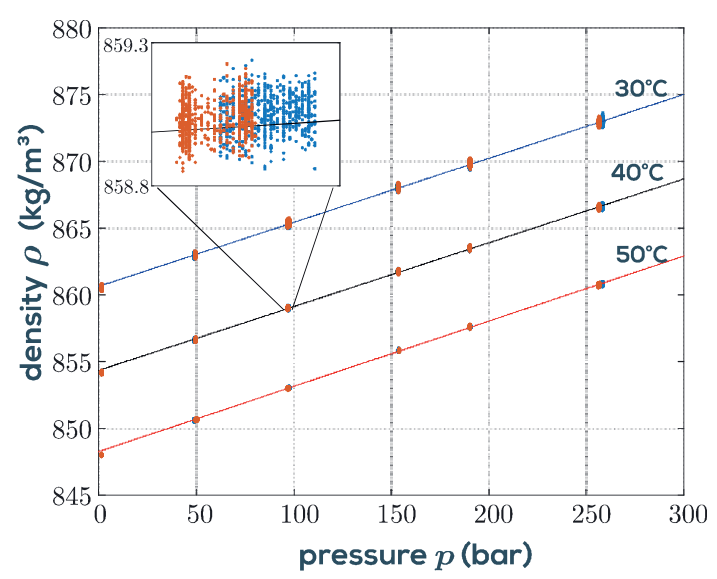

Fig. 7: Density of hydraulic oil over pressure. at three different temperatures, simultaneously measured with viscosity in Fig. 6.
While temperature sweeps can directly be performed by the measurement cell, for pressure variation an external pressure regulator is required. Small variations in temperature $(\Delta T)$ and pressure $(\Delta p)$ also cause a change in density

$$
\frac{\Delta \rho}{\rho} \approx \underbrace{\frac{1}{\rho}\left(\frac{\partial \rho}{\partial p}\right)_{T}}_{\beta_{T}} \Delta p+\underbrace{\frac{1}{\rho}\left(\frac{\partial \rho}{\partial T}\right)_{p}}_{-\gamma_{p}} \Delta T
$$

The two additional monitoring parameters in Eq. (3) are the isothermal compressibility $\beta_{T}$ and the isobaric heat expansion coefficient $\gamma_{p}$. The inverse of the isothermal compressibility is also known as the isothermal bulk modulus $K_{T}$. In Tab. 1, the approximate ranges of bulk modules for three pressure fluids (from [12]) are listed.

Tab. 2: The range of the bulk modulus $K_{T}$ for oils measured [12].

\begin{tabular}{|c|l|}
\hline$(1.4 \ldots 1.6) \cdot 10^{4}$ & air-free mineral oil \\
\hline$(1.0 \ldots 1.2) \cdot 10^{4}$ & mineral oil with air \\
\hline$(2.3 \ldots 3.5) \cdot 10^{4}$ & $\begin{array}{l}\text { non-mineralic high-pres- } \\
\text { sure fluids. }\end{array}$ \\
\hline
\end{tabular}

In Tab. 3 and 4 these coefficients derived from the measurements in Fig. 7 are shown. It has to be emphasized that the derivatives in Eq. (3), are sensitive to the fitting model. It is therefore advisable to record more temperature points than are shown in Fig. 4. Due to the short thermal time constants of the measurement cell of around 1-2 minutes per temperature point, this is not a limiting factor.

As indicated in Tab 1, the amount of dissolved air can be estimated on basis of the bulk modulus. Its measurement is therefore of interest for hydraulic system engineers. In this field three approaches for measuring the bulk modulus are commonly used [13]: i) the volume change method, ii) the flow change method and iii) sound wave propagation. Measurement of the bulk modulus by evaluating the local density variation using the micro sensor has certain advantages over these methods. In the volume change method (i), where the reduction of a defined volume is measured versus applied pressure, or for the flow change method (ii) where a small volume is injected into the chamber and pressure is measured, the finite rigidity of the chamber introduces errors. Furthermore, measurements are meaningful only at high pressures where all entrapped air bubbles are dissolved [14]. The technical realizations of these principles are therefore generally complicated, 
expensive and require significant calibration efforts [15]. Due to the larger observed volumes, sustaining thermal equilibrium in the entire volume is also more demanding. From acoustic propagation measurements (iii) the speed of sound and consequently the adiabatic bulk modulus $K_{A}$ can be determined using Eq. (4).

$$
K_{A}=c^{2} \rho
$$

It is emphasized that the adiabatic bulk modulus $K_{S}$ differs from $K_{T}$ by ca. $10-20 \%$ for mineral oil. They can be interrelated, but specific heat capacitance, heat expansion coefficient, density and temperature are required [15]. Nevertheless, speed of sound is a parameter of interest on its own, because it characterizes pressure wave propagation in high speed switching hydraulics [16].

Tab. 3 isobaric heat expansion coefficient at $\vartheta=40^{\circ} \mathrm{C}$ for different pressures

\begin{tabular}{|l|l|l|l|l|}
\hline$p$ (bar) & 1 & 50 & 100 & 190 \\
\hline$\gamma_{p}\left(10^{-4} \mathrm{~K}^{-1}\right)$ & 7.34 & 7.19 & 7.1 & 7.095 \\
\hline
\end{tabular}

Tab. 4 isothermal compressibility and bulk modulus at $p=100$ bar for different temperatures.

\begin{tabular}{|l|l|l|l|}
\hline$\vartheta\left({ }^{\circ} \mathrm{C}\right)$ & $30^{\circ} \mathrm{C}$ & $40^{\circ} \mathrm{C}$ & $50^{\circ} \mathrm{C}$ \\
\hline$\beta_{T}\left(\mathrm{GPa}^{-1}\right)$ & 0.565 & 0.575 & 0.588 \\
\hline$K_{\mathrm{T}}(\mathrm{GPa})$ & 1.77 & 1.74 & 1.70 \\
\hline
\end{tabular}

\section{Oil condition monitoring in automo- tive applications.}

The diesel particulate filters (PDF) used in vehicles require periodic regeneration to prevent clogging. For this purpose, one approach is by exhausting late post-injected unburnt diesel is to the filter to burn off the accumulated soot. Oil dilution occurs when part of the late injected fuel is wiped into the crankcase by the piston rings and thus into the engine oil (see Fig. 8). The addition of biodiesel aggravates this issue, because the boiling point of biodiesel is higher than that of conventional diesel [17]. Therefore, biodiesel evaporates at the cylinder walls at a lower rate. Furthermore, biodiesel has strong chemical interactions with various additives in engine oil and thus hinders, for example, the cleaning effect of the oil. Therefore, there are efforts by engine developers to minimize fuel entry by optimizing late-injection schemes. This happens, for example, by adjustments of the number and duration of the individual injections, as is shown in 5 . In order to be able to assess the effect of different settings faster than by using off-site laboratory analyzes of oil samples, an online monitoring system measuring viscosity and density can be used.

Although, the densities of diesel and engine oil are very similar, the viscosities are distinctly different. While diesel-addition lowers the viscosity of the oil, it is increased by an unavoidable entry of combustion residues (soot).

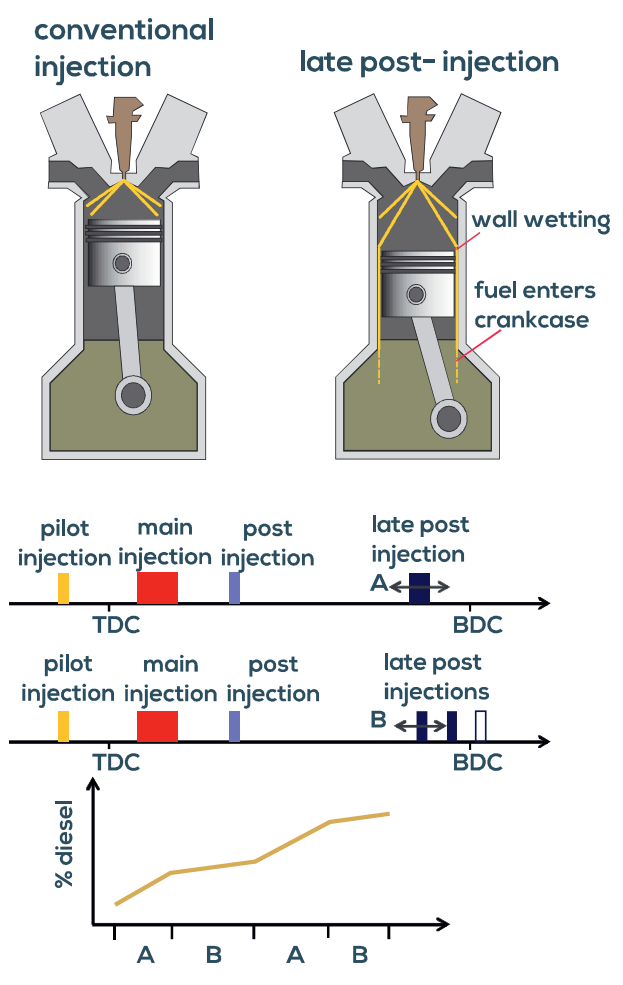

Fig. 8: Late post injections for particle filter regeneration causes gradual oil dilution [17]. Optimization of injection timings can reduce this effect, but requires close monitoring of diesel content.

It is therefore required to separate the effects of soot and diesel which requires an additional parameter to be measured. As soot increases the density of the oil, it is possible to calculate the diesel and soot content by simultaneously measuring density and viscosity. Fig. 9 shows the effects of diesel and soot entry into engine oil for two different operating cycles $A$ and $B$. Fig. 10 shows the test setup, which is used to assess the general performance of the measurement system. For the experiment, an oil sample of $200 \mathrm{ml}$ was prepared consisting of $50 \mathrm{ml}$ used oil (containing $5 \%$ diesel and an unknown soot content) and $150 \mathrm{ml}$ new oil of the 
same batch. The overall diesel content is therefore $1 \%$ and the soot content is 0.2 used-oil-soot equivalents. Computer controlled syringe pumps add two mixtures $A$ and $B$ at a rate of $100 \mu \mathrm{l} / \mathrm{min}$ to the mixture which is continuously stirred to guarantee homogeneity. A sample is pumped into the measurement cell in intervals of 8 minutes and measurements are taken after reaching thermal equilibrium.
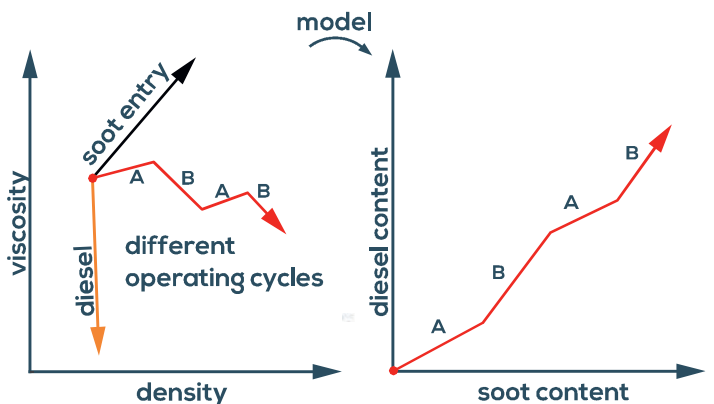

Fig. 9: Diesel and soot have different effects on viscosity and density and can therefore be calculated by a mixture model.

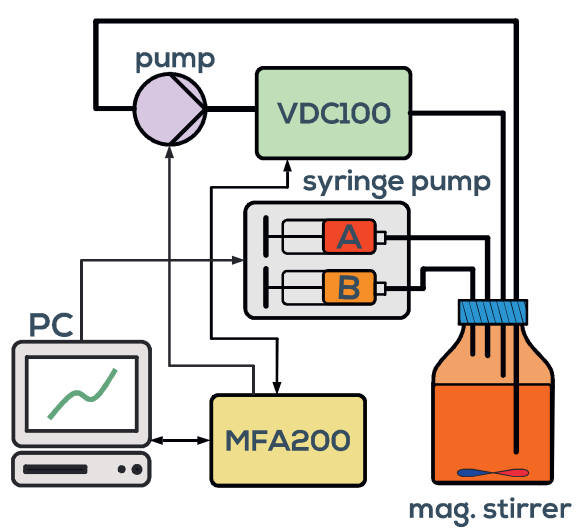

Fig. 10: Schematic of the laboratory test setup used to asses to achievable performance of the system.

The concentrations are therefore known throughout the experiment and can be compared with the estimated values in order to assess the achievable performance. Fig. 11 and Fig. 12 show viscosity and density. A linearized mixing model, valid for low foreign substance concentrations is used to determine the diesel and used-oil soot equivalents in Fig. 13 and Fig. 14 , respectively. The entry of substances is stopped for approximately $3 \mathrm{~h}$ indicated by the plateaus. No significant drifts are observed and the actual diesel and soot equivalents are shown as dashed lines. The agreement and sensitivity of the system suggests that the system is suitable for this task and reaching an accuracy of $0.1 \%$ diesel content is feasible.

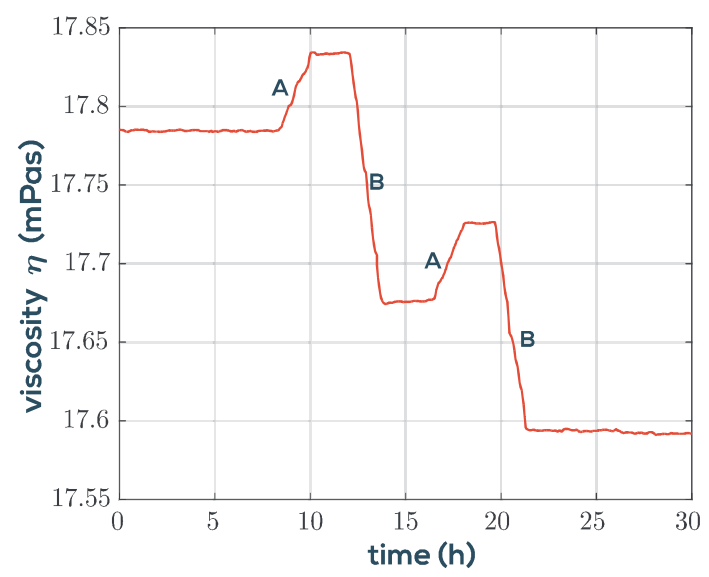

Fig. 11: Viscosity vs. time obtained with the setup in Fig. 10.

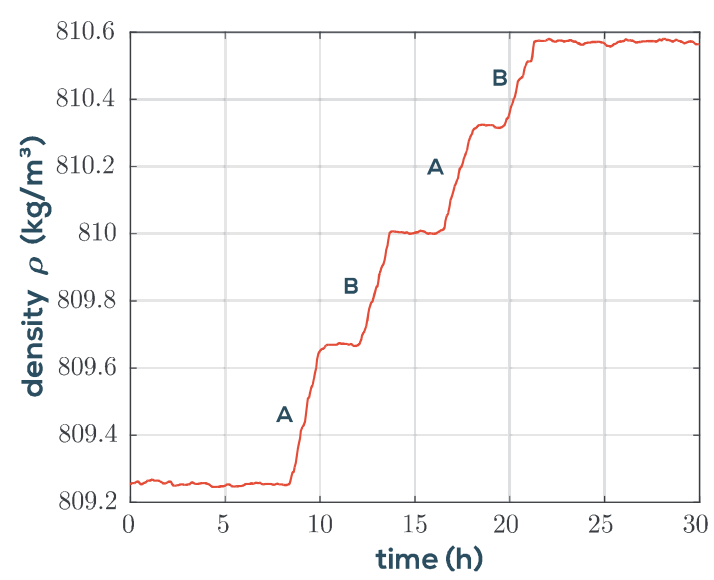

Fig. 12: Density vs. time.

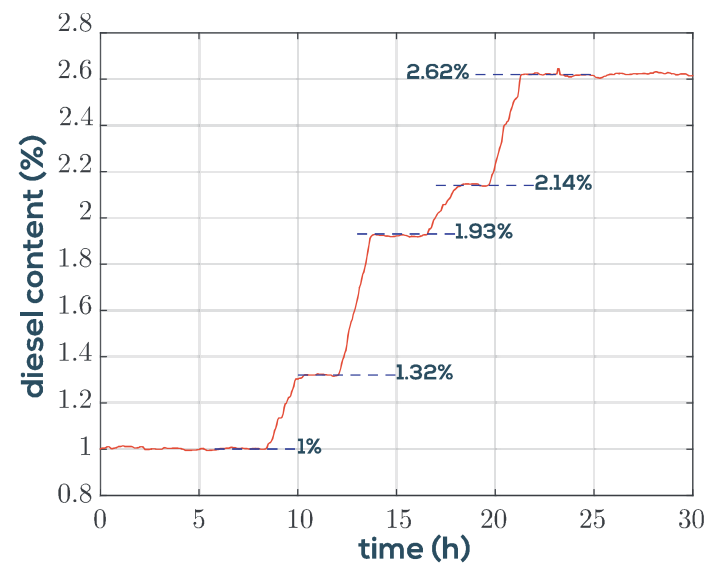

Fig. 13: Measured diesel content vs. time. The dashed lines represent the real values known throughout the experiment. 


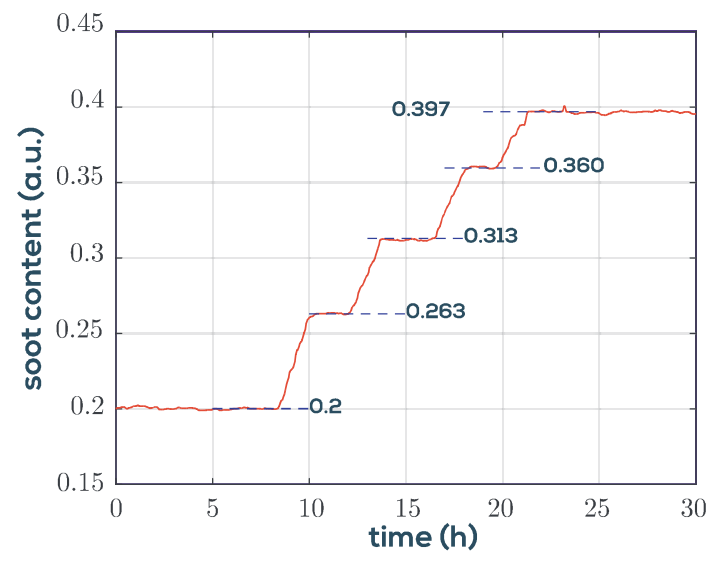

Fig. 14: Soot equivalents vs time. The dashed lines represent the real values.

\section{Conclusions}

The ability of the described online condition monitoring system to measure viscosity and density over a large temperature and pressure range yields additional monitoring parameters such as the bulk modulus, the heat expansion coefficient, but also the V-I index and the pressure-viscosity functions. These parameters are for instance relevant for the determination of aging of lubricating oils or dissolved air in hydraulic systems. Furthermore, the applicability of the system to measure diesel and soot concentrations in engine oil was demonstrated on basis of simultaneous viscosity and density sensing. The results indicate high sensitivity and accuracy.

\section{References}

[1] A. Davies, Handbook of Condition Monitoring: Techniques and Methodology, Springer, 1989 doi: 10.1007/978-94-011-4924-2

[2] M. Blanke et.al., Diagnosis and Fault-Tolerant Control, 3rd Edition, Springer, (2016) doi: 10.1007/978-3-662-47943-8

[3] A.O. Niedermayer, T. Voglhuber-Brunnmaier, M. Heinisch, B. Jakoby, Accurate Determination of Viscosity and Mass Density of Fluids using a Piezoelectric Tuning Fork Resonant Sensor, Proceedings SENSOR 66-71. (2015), doi: 10.5162/sensor2015/A2.4

[4] B. Jakoby, M. J. Vellekoop, Viscosity sensing using a Love-wave device. Sensors and Actuators A: Physical, 68(1-3), 275-281 (1998) doi: 10.1016/S0924-4247(98)00017-X

[5] L. Matsiev, J. Bennett, E. McFarland, Application of low frequency mechanical resonators to liquid property measurements. IEEE Ultrasonics Symposium. Proceedings IEEE, 1, 459-462. (1998) doi: 10.1109/ULTSYM.1998.762188
[6] B. Jakoby, R. Beigelbeck, F. Keplinger, F. Lucklum, A.O. Niedermayer, ... B. Weiss, Miniaturized sensors for the viscosity and density of liquids-performance and issues. IEEE transactions on ultrasonics, ferroelectrics, and frequency control, 57(1), 111-120. (2010) doi: 10.1109/TUFFC.2010.1386

[7] T. Voglhuber-Brunnmaier, E. K. Reichel, E. K., A. O. Niedermayer, F. Feichtinger, J.K. Sell, B. Jakoby, Determination of particle distributions from sedimentation measurements using a piezoelectric tuning fork sensor. Sensors and Actuators A: Physical, 284, 266-275. (2018), doi: 10.1016/j.sna.2018.10.038

[8] B. Jakoby, M. J. Vellekoop, Physical sensors for water-in-oil emulsions. Sensors and Actuators A: Physical, 110, (1-3), 28-32. (2004). doi: 10.1016/j.sna.2003.08.005

[9] S. J. Martin, K. O. Wessendorf, C. T. Gebert, G. C. Frye, R. W. Cernosek, L. Casaus, M. A. Mitchell, Measuring liquid properties with smooth-and textured-surface resonators. IEEE international frequency control symposium, 603608. (1993), doi: 10.1109/FREQ.1993.367450

[10] A.O. Niedermayer, T. Voglhuber-Brunnmaier, F. Feichtinger, M. Heinisch, B. Jakoby, Online Condition Monitoring of Lubricating Oil based on Resonant Measurement of Fluid Properties. Sensors and Measuring Systems; 19th ITG/GMA-Symposium VDE. 1-4 (2018), isbn: 978-3-8007-4683-5

[11] T. Voglhuber-Brunnmaier, A.O. Niedermayer, F. Feichtinger, M. Heinisch, B. Jakoby, High Precision Resonant Sensor Evaluation with Application to Fluid Sensing, Sensors and Measuring Systems. Sensors and Measuring Systems; 19th ITG/GMA-Symposium VDE. 355-358 (2018), isbn: 978-3-8007-4683-5

[12] D. Will, N. Gebhardt, (Eds.). Hydraulik: Grundlagen, Komponenten, Systeme. Springer-Verlag. (2015), doi: 10.1007/978-3-662-44402-3

[13] C. Ding, C., H. Murrenhoff, Accuracy estimation of a novel setup for online bulk modulus measurement in hydraulic systems. O+P Ölhydraulik und Pneumatik 3, (2016)

[14] J. Wang, G. Gong, H. Yang, Control of bulk modulus of oil in hydraulic systems. IEEE/ASME International Conference on Advanced Intelligent Mechatronics, 1390-1395 (2008), doi: 10.1109/AIM.2008.4601865

[15] R. Haas, Material behavior of hydraulic fluids under the influence of thermoelasticity and entrained air. Trauner. (2013), isbn: 978-3990331774

[16] R. Scheidl , H. Kogler, B. Winkler, Hydraulic switching control-objectives, concepts, challenges and potential applications. Hidraulica, 1, (2013), issn: 1453-7303

[17] D. Uy, R. J. Zdrodowski, A. E. O'Neill, S. J. Simko, A. K. Gangopadhyay, M. Morcos, ... \& G. Parsons. Comparison of the effects of biodiesel and mineral diesel fuel dilution on aged engine oil properties. Tribology Transactions, 54(5), 749-763. (2011), doi: $10.1080 / 10402004.2011 .597545$ 